6. Kojima H, Nojima T, Nagashima K, et al. Diabetes insipidus caused by lymphocytic infundibuloneurohypophysitis. Arch Pathol Lab Med 1989; 113: 1399-1401.

7. Panicker HK, Janicic N, Nguyen D, Verbalis J. Presumed infundibuloneurohypophysitis: unusual presentation in a postpartum patient. Am J Neuroradiol 2005; 26: 357-9.

8. Kanou Y, Arita K, Kurisu K, Tominaga A, Akimitsu T. Infundibuloneurohypophysitis presenting a large sellarjuxtasellar mass: case report. Surg Neurol 2004; 61: 278-81.

9. Amagasa M, Yuda F, Kojima H, Noshita N, Sato S. Natural course of lymphocytic infundibuloneurohypophysitis. Clin Neuropathol 2001; 20: 229-32.

10. Hashimoto K, Takao T, Makino S. Lymphocytic adenohypophysitis and lymphocytic infundibuloneurohypophysitis. Endocr J 1997; 44: $1-10$

11. Takao T, Asaba K, Tanaka H, Matsumoto R, Nanamiya W, Hashimoto $\mathrm{K}$. A case of Iymphocytic infundibuloneurohypophysitis showing diabetes insipidus followed by anterior hypopituitarism associated with thrombasthenia. Endocr J 2000; 47: 285-91.

\section{Utilización de internet por los pacientes de oncología}

\section{Sr. Director:}

El desarrollo de la informática y de Internet han supuesto una auténtica revolución en el desarrollo de la medicina (1).

El acceso a las bases de datos, el intercambio de información entre los profesionales y las búsquedas bibliográficas son algunas de las múltiples ventajas que internet nos aporta (2).

Sin embargo, somos menos conscientes de que nuestros pacientes también pueden utilizar la red con la finalidad de consultar sobre su enfermedad e incluso sobre si las pruebas diagnósticas o el tratamiento propuesto se ajusta a la práctica médica habitual en hospitales de alto nivel (3).

Se ha analizado el uso de Internet en pacientes con enfermedades endocrinológicas, digestivas y reumáticas y en dichas patologías entre el 18 y el $44 \%$ de los pacientes habían utilizado Internet para buscar información médica sobre su enfermedad (4-6).

Decidimos efectuar un pequeño estudio en el que tratábamos de analizar la frecuencia con la que los enfermos atendidos en la consulta de oncología de nuestro hospital utilizaban internet para buscar información sobre su enfermedad.

Se realizó una encuesta, modificada con respecto a la ya empleada en un estudio similar realizado en una unidad de enfermedades autoinmunes de nuestro país (4); en la que se recogían los siguientes datos: a) edad y sexo; b) tipo de estudios que ha realizado: Ninguno, elemental, bachiller y superior; c) ¿ha buscado información sobre su enfermedad en Internet?; d) ha buscado información sobre su enfermedad por otros medios: prensa, enciclopedia médica, amigos u otros médicos; y e) ¿le gustaría que el médico le facilitará direcciones de internet, donde consultar sobre su enfermedad?

Se recogieron 30 formularios cumplimentados de la encuesta sobre los 45 entregados. A todos los pacientes se les preguntó si sabían leer y escribir y únicamente en el caso positivo se les daba el formulario. Tampoco se entregó la encuesta a los pacientes de edad muy avanzada, al estimar que habrían tenido escaso contacto con las últimas tecnologías.

Con respecto a los resultados del estudio, la edad media era de 58 años, y existía un predominio de varones (18-60\%).
El tipo de estudios que habían realizado fue elemental en 8 (26\%), bachiller en $16(53 \%)$ y superior en $6(20 \%)$.

Referían haber buscado información por internet 6 pacientes $(20 \%)$ y por otros medios otros 6 casos $(20 \%)$, habitualmente por amigos y otros médicos.

La edad media de los pacientes que habían utilizado Internet fue de 42 años, respecto a los 58 años de la edad media del grupo, por lo que vemos notablemente menor.

12 pacientes indicaron que les gustaría que su médico les facilitará direcciones de Internet para consultar sobre su enfermedad (40\%). En este grupo de pacientes se incluían los 6 que reconocían que ya habían buscado anteriormente información por internet sobre su enfermedad y además otros seis, que nunca antes lo habían realizado, pero que les gustaría realizarlo en el futuro.

Analizando el tipo de estudios que habían cursado los pacientes que usaban internet, 5 de los 6 habían cursado estudios de nivel superior y uno bachiller y de los que desearían que su médico les facilitará direcciones por Internet útiles para conocer más sobre su enfermedad, 5 habían realizado estudios de nivel superior y otros 5 de bachiller.

Como podemos apreciar a mayor nivel de estudios, mayor es la probabilidad de que una persona buscara información adicional en la red, el mismo resultado se ha encontrado anteriormente en otros estudios $(6,7)$, así como también que a menor edad se consulta la red con una mayor frecuencia, lo cuál parece lógico, al tratarse de una técnica con desarrollo en los últimos años $(3,7)$.

Resulta evidente que la utilización de la red será cada vez mayor en los próximos años, además diversos estudios han evidenciado que los pacientes mejor informados cumplen con una mayor adherencia las indicaciones de su médico. Por otra parte, la opinión de los enfermos tiene cada vez un mayor peso en la decisión final sobre qué tratamiento realizar en ese paciente concreto.

Lamentablemente internet no es sinónimo de calidad, dado que hay muchas páginas de una calidad muy deficiente, por lo que sería importante que el médico participará en la selección de las páginas web para su paciente y también en la elaboración de páginas informativas de calidad a través de las diferentes sociedades médicas y de los grupos de trabajo.

\section{F. Marcos Sánchez, M. I. Albo Castaño, A. Viana Alonso, A. Moyano Jato}

Servicio de Medicina Interna. Sección de Oncología. Hospital Nuestra Señora del Prado. Talavera de la Reina. Toledo

1. Kassirer JP. The next transformation in the delivery of health care. N Eng J Med 1995; 332: 52-54

2. Armstrong R. Appropriate and effective use of the Internet and databases. Clin Rheumatol 2003; 22: 173-176.

3. Smith-Barbaro PA, Licciardone JC, Clarke HF, Coleridge ST. Factors associated with intended use of a Web site among family practice patients. J Med Internet Res 2001; 3: E17.

4. Ortega-Centeno N, Callejas Rubio JL, Benticuaga Martínez MN, Navarro Pelayo F, de la Higuera Torres-Puchol J. Utilización de Internet como fuente de información por los pacientes de una consulta de Enfermedades Autoinmunes sistémicas. Rev Clin Esp 205; 205: 25-258.

5. O'Connor B, Johanson JF. Use of the Web for medical information by a gastroenterology clinic population. JAMA 2000; 284: 1962-1964.

6. Panes J, de Lacy AM, Sans M, Soriano A, Pique JM. Frequent Internet use among Catalan patients with inflammatory bowel disease. Gastroenterol Hepatol 2002; 25: 306-309.

7. Tassone P, Georgalas C, Patel NN, Appleby E, Kotecha B. Do otolaryngology out-patients use the internet prior to attending their appointment? J Laryngol Otol 2004; 118: 34-38. 